\title{
The Mathematical Foundations of Dynamic User Equilibrium
}

\author{
Terry L. Friesz ${ }^{\mathrm{a}}, \mathrm{Ke} \mathrm{Han}^{\mathrm{b}, *}$ \\ ${ }^{a}$ Department of Industrial and Manufacturing Engineering, Pennsylvania State University, USA. \\ ${ }^{b}$ Department of Civil and Environmental Engineering, Imperial College London, United Kingdom.
}

\begin{abstract}
This paper is pedagogic in nature, meant to provide researchers a single reference for learning how to apply the emerging literature on differential variational inequalities to the study of dynamic traffic assignment problems that are Cournot-like noncooperative games. The paper is presented in a style that makes it accessible to the widest possible audience. In particular, we apply the theory of differential variational inequalities (DVIs) to the dynamic user equilibrium (DUE) problem. We show that there is a DVI whose necessary conditions describe a DUE. We restate the flow conservation constraint associated with each origin-destination pair as a first-order two-point boundary value problem, thereby leading to a DVI representation of DUE; then we employ Pontryagin-type optimality conditions to show that any DVI solution is a DUE. We also show that the DVI formulation leads directly to a fixed-point algorithm. We explain the fixed-point algorithm by showing the calculations intrinsic to each of its steps when applied to simple examples.
\end{abstract}

Keywords:

dynamic user equilibrium, differential variational inequality, optimal control, fixed point algorithm

\section{Introduction}

The question of how to mathematically formulate the dynamic user equilibrium problem has been discussed in considerable depth in the transportation research literature. Some of the many relevant works are Friesz et al. (1993, 2001); Lo and Szeto (2002); Bliemer and Bovy (2003); Szeto and Lo (2004); Ban et al. (2008); Friesz et al. (2011); Ban et al. (2012); Friesz et al. (2013); Friesz and Meimand (2014); Han et al. (2015a,b). For the most part these works put forward some sort of variational inequality in discrete or continuous time, and each such formulation is either a differential variational inequality or can be reformulated as a DVI. Unlike some other expository papers on dynamic traffic assignment (Peeta and Ziliaskopoulos, 2001; Szeto and Lo, 2005; Chiu et al., 2011), this paper is an informal primer on using differential variational inequality theory as the foundation for dynamic user equilibrium. This has been done to help what some see as a very large barrier to understanding dynamic user equilibrium, when approached from the perspective of DVIs. A knowledge of elementary calculus and the willingness to work though some complicated algebra is all that should be needed for one to profit from reading this paper. It is hoped that this paper will facilitate mastering the essentials of differential variational inequality theory, and encourage its use in crafting new models and algorithms within the field of inquiry known as transportation science.

\section{Discrete and Continuous Time}

It is often argued that, since all dynamic models must ultimately be solved by discretizing the time horizon of interest, it is a waste of time to develop continuous time models. The premise of this assertion is false, because, as we show in this paper, continuous time models may be solved in continuous time. Moreover, continuous time may be used to describe fast subsystems of the system being studied, while slower subsystems may be modeled with discrete

\footnotetext{
${ }^{*}$ Corresponding author

Email addresses: tfriesz@psu.edu (Terry L. Friesz), k.han@imperial .ac.uk (Ke Han )
} 
time. Such a model is naturally and innately a dual-timescale model, and it may be formed and solved using the ideas put forth in this paper. Moreover, continuous time solution of a continuous time model does not mean one must be able to find a closed-form solution; we will see that iterative algorithms that produce progressively more accurate continuous-time solutions may be easily devised and implemented.

It is also the case that continuous-time models may often be discretized and solved with discrete time algorithms. From this perspective, continuous-time formulations are a shorthand for model developers, who can, after the formulation stage is complete, discretize and prepare a model for numerical solution with finite-dimensional algorithms. Also, the reader should take care not to overlook in the technical material ahead that the differential equations for primal and dual variables, generated at each step of the algorithmic approach we stress herein, have righthand sides that are functions of time only. As such, the primal and dual dynamics may be solved trivially, in closed form if polynomial representations of the righthand sides are employed.

Furthermore, it is important to note that DUE modeling, at its most fundamental level, intrinsically involves flow-propagation constraints (FPCs) as part of the dynamic network loading (DNL) process that must be executed whenever the value of path delay is needed. Such constraints ensure flow entering an arc equals flow exiting that same arc at a future time. Discrete-time models involving such time shifts are intrinsically difficult to form and even more difficult to solve. In discrete time, FPCs require time steps of unequal length, fractional time steps, or some other approximation. For continuous time, the presence of FPCs, may be handled straightforwardly. This is important if one "unfolds" the DNL model used in conjunction with a DUE model, whereby the FPCs become explicit constraints in the DUE calculation as in Friesz et al. (2001).

\section{History of the DVI Formalism}

It was within the transportation modeling literature that the modern DVI formalism was first introduced by Friesz et al. (2004) and Pang and Stwart (2008), although studies emerged earlier in the optimization literature, which are closely related to the development of DVIs (Hipfel, 1993; Heemels, 1999; Camlibel, 2001). A DVI is, crudely speaking, an optimal control problem with its single objective replaced by a variational inequality. The adjective "differential" arises because in optimal control theory the constraints contain explicit state dynamics, as well as constraints on the control variables. It should be noted that an infinite dimensional variational inequality is generally not a DVI. That is, a VI in a Hilbert space, or more generally in a Banach space, is not a DVI unless it has explicit dynamics (differential or difference equations) that influence its solution. Although some papers initially used the name VICON for variational inequalities with control variables and explicit state dynamics, most scholars presently use "DVI" in the same sense we do herein. The DVI formalism builds on the transportation-related work of Friesz et al. (1993); Bernstein et al. (1993) and Friesz et al. (2001). This early literature used formulations that are special cases of the DVI problem. The DVI formalism now widely employed originates with Friesz et al. (2004), and includes Friesz and Mookherjee (2006), Friesz et al. (2006), Friesz et al. (2007a), and Friesz et al. (2007b). These papers formally present abstract differential variational inequalities, and illustrate how DVIs may be employed in transportation and regional science applications. Subsequently, Pang and Stwart (2008) provided a broader survey of alternative formulations and applicable numerical solution techniques. Pang and Stwart (2008) refer to their work as the first formal introduction of differential variational inequalities (DVIs). That claim notwithstanding, the first statement of the modern differential variational (DVI) problem appears in Friesz et al. (2004). Two other publications supporting this reading of the literature are Bernstein et al. (1993) and Friesz et al. (2007b). It is within the transportation modeling literature that the modern DVI formalism was first introduced. In particular, Bernstein et al. (1993) present a DVI for one version of DUE. Their formulation is the first DVI reported in either the transportation or game theory literature; it employs the link delay model for network loading. Friesz et al. (2004) provide the first statement of an abstract DVI. Subsequently, Pang and Stwart (2008) catalog various types of DVIs, along with results on existence, uniqueness, and algorithm convergence (Camlibel et al., 2006a,b, 2008). Contemporaneously with the review by Pang and Stwart (2008), Mookherjee and Friesz (2008) and Friesz et al. (2008) illustrated the importance of DVIs to service sector economic analysis, urban logistics, and competitive supply chain modeling.

The application of concern in this paper is the dynamic user equilibrium (DUE) problem at the core of dynamic traffic assignment (DTA). Most published mathematical formulations of DUE express an open-loop version of a dynamic notion of user equilibrium (DUE) based on some type of generalization of Wardrop's so-called first principle. Presently some DUE models treat both route choice and departure time choice as fundamental decisions, while others 
are concerned with either departure time choice or route choice, but not both. In this paper we are concerned only with simultaneous route and departure time choice, abbreviated as 'SRDT choice' or simply 'SRDT' when no confusion will arise. As the works cited in Section 1 explain, dynamic user equilibrium (DUE) assumes that some type of dynamic network loading operator, abbreviated as 'DNL operator' or simply 'DNL' when no confusion will arise, is available for each path, although there is no need for the underlying DNL model to have closed-form solutions. The DNL operator provides the effective path delay for every path considered. There are a few different ways a SRDT DUE may be defined. Most definitions of SRDT DUE are equivalent.

\section{Notation and Elementary Relationships}

Before we introduce variational inequality formulations for dynamic user equilibria, we will first provide some definitions and terminology required to describe the notion of a DUE. The time interval of analysis is a single commuting period or "day" expressed as $\left[t_{0}, t_{f}\right] \subset \mathbb{R}$ where $\mathbb{R}$ denotes the set of real numbers and $t_{0}<t_{f}$. We let $\mathcal{P}$ be the set of all paths employed by travelers. For each path $p \in \mathcal{P}$ we define the path departure rate, which is a function of departure time $t \in\left[t_{0}, t_{f}\right]$ :

$$
h_{p}(\cdot):\left[t_{0}, t_{f}\right] \rightarrow \mathbb{R}_{+},
$$

where $\mathbb{R}_{+}$denotes the set of non-negative real numbers. Each path departure rate (path flow) $h_{p}(t)$ is interpreted as a time-varying quantity, measured at the entrance of the first arc of the relevant path, and the units for the path flows are usually vehicles per unit time. We next define $h(\cdot)=\left\{h_{p}(\cdot): p \in \mathcal{P}\right\}$ to be a vector of departure rates (path flows). Without creating confusion, we use $h(\cdot)$ or simply $h$ to represent the vector-valued function of $t$, the departure time.

We denote the space of square-integrable functions on the interval $\left[t_{0}, t_{f}\right]$ by $L^{2}\left[t_{0}, t_{f}\right]$, and its subset consisting of non-negative functions by $L_{+}^{2}\left[t_{0}, t_{f}\right]$. We stipulate that each path flow is square integrable; so that

$$
h_{p}(\cdot) \in L_{+}^{2}\left[t_{0}, t_{f}\right], \quad h(\cdot) \in\left(L_{+}^{2}\left[t_{0}, t_{f}\right]\right)^{|\mathcal{P}|},
$$

where $\left(L_{+}^{2}\left[t_{0}, t_{f}\right]\right)^{|\mathcal{P}|}$ is a cone ${ }^{1}$ of the $|\mathcal{P}|$-fold product space $\left(L^{2}\left[t_{0}, t_{f}\right]\right)^{|\mathcal{P}|}$ consisting of non-negative vector-valued functions.

We note that, by the measure-theoretic nature of the DUE model (Friesz et al., 1993), the path departure rates $h_{p}(\cdot), p \in \mathcal{P}$, are defined only up to a set of measure zero. Letting $v$ be a Lebesgue measure on $\left[t_{0}, t_{f}\right]$, one should write $\forall_{v}\left(t \in\left[t_{0}, t_{f}\right]\right)$ to represent the phrase for $v$-almost all $t \in\left[t_{0}, t_{f}\right]$ or simply write $\forall_{v}(t)$. In other cases, we may suppress any reference to $v$-almost all $t \in S$ so long as doing so does not create confusion.

In DUE modeling, the single most crucial ingredient is the path delay operator, which maps a given vector of departure rates $h$ to a vector of path travel times. More specifically, we let

$$
D_{p}(t, h) \quad \forall_{v}\left(t \in\left[t_{0}, t_{f}\right]\right), \quad \forall p \in \mathcal{P}
$$

be the path travel time of a driver departing at time $t$ and following path $p$, given the departure rates associated with all the paths in the network, which are expressed by $h$ immediately above. We then define the path delay operator $D(\cdot)$ by letting $D(h)=\left\{D_{p}(\cdot, h): p \in \mathcal{P}\right\}$, which is a vector of time-dependent path travel times $D_{p}(t, h)$. We use $D(\cdot)$ to denote an operator defined on $\left(L_{+}^{2}\left[t_{0}, t_{f}\right]\right)^{|\mathcal{P}|}$, which maps a vector valued function $h(\cdot)$ to another vector-valued function $\left\{D_{p}(\cdot, h): p \in \mathcal{P}\right\}$. More precisely,

$$
\begin{gathered}
D(\cdot):\left(L_{+}^{2}\left[t_{0}, t_{f}\right]\right)^{|\mathcal{P}|} \longrightarrow\left(L_{+}^{2}\left[t_{0}, t_{f}\right]\right)^{|\mathcal{P}|} \\
h(\cdot)=\left\{h_{p}(\cdot), p \in \mathcal{P}\right\} \longrightarrow D(h)=\left\{D_{p}(\cdot, h), p \in \mathcal{P}\right\}
\end{gathered}
$$

The effective delay operator $\Psi$ is similarly defined, except that the effective path delay contains, in addition to path travel time, the pertinent arrival penalties. Thus, the effective path delay is a more general notion of "travel cost" than

\footnotetext{
${ }^{1} \mathrm{~A}$ cone in a vector space is a subset that is closed under multiplication by positive scalars,
} 
path delay. The effective delay operator is defined as follows.

$$
\begin{gathered}
\Psi:\left(L_{+}^{2}\left[t_{0}, t_{f}\right]\right)^{|\mathcal{P}|} \rightarrow\left(L_{+}^{2}\left[t_{0}, t_{f}\right]\right)^{|\mathcal{P}|} \\
h(\cdot)=\left\{h_{p}(\cdot), p \in \mathcal{P}\right\} \mapsto \Psi(h)=\left\{\Psi_{p}(\cdot, h), p \in \mathcal{P}\right\}
\end{gathered}
$$

where

$$
\Psi_{p}(t, h)=D_{p}(t, h)+f\left(t+D_{p}(t, h)-T_{A}\right) \quad \forall t \in\left[t_{0}, t_{f}\right], \quad \forall p \in \mathcal{P}
$$

when $T_{A}$ is the desired arrival time and $T_{A}<t_{f}$. The term $f\left(t+D_{p}(t, h)-T_{A}\right)$ assesses a nonnegative penalty whenever

$$
t+D_{p}(t, h) \neq T_{A}
$$

since $t+D_{p}(t, h)$ is the clock time at which departing traffic arrives at the destination of path $p \in \mathcal{P}$. The arrival penalty function $f(\cdot)$ usually assumes a ' $\mathrm{V}$ ' shape; see, for example, (7.33). Note that, for convenience, $T_{A}$ is assumed to be independent of destination. However, that assumption is easy to relax, and the consequent generalization of our model is a trivial extension.

We interpret $\Psi_{p}(t, h)$ as the perceived travel cost of drivers departing at time $t$ following path $p$ given the vector of path departure rates $h$. We stipulate that each path effective delay

$$
\Psi_{p}(\cdot, h):\left[t_{0}, t_{f}\right] \longrightarrow \mathbb{R}_{++} \quad \forall p \in \mathcal{P}
$$

is measurable, strictly positive, and square integrable, where $\mathbb{R}_{++}$denotes the set of positive real numbers. The notion of strictly positive functions, as we employ them throughout this paper, refers to measurable functions that are positive almost everywhere. The notation

$$
\Psi(h) \doteq\left\{\Psi_{p}(\cdot, h): p \in \mathcal{P}\right\} \in\left(L_{+}^{2}\left[t_{0}, t_{f}\right]\right)^{|\mathcal{P}|}
$$

is used to express the complete vector of effective delays.

In order to define an appropriate concept of minimum travel costs in the present context, we require the measuretheoretic analog of the infimum of a set of numbers. In particular, for any measurable function $g(\cdot):\left[t_{0}, t_{f}\right] \rightarrow \mathbb{R}$, the essential infimum of $g(\cdot)$ on $\left[t_{0}, t_{f}\right]$ is given by

$$
\underset{s \in\left[t_{0}, t_{f}\right]}{\operatorname{essinf}}\{g(s)\} \doteq \sup \left\{x \in \mathbb{R}: v\left\{s \in\left[t_{0}, t_{f}\right]: g(s)<x\right\}=0\right\}
$$

where $v$ is a Lebesgue measure. Note that for each $x>\underset{s \in\left[t_{0}, t_{f}\right]}{\operatorname{essinf}}\{g(s)\}$ it must be true by definition that

$$
v\left\{s \in\left[t_{0}, t_{f}\right]: g(s)<x\right\}>0
$$

In other words, the essential infimum $\hat{g}$ of $g(\cdot)$ is the largest number such that $g(\cdot)$ is no less than $\hat{g}$ almost everywhere. Let us define the essential infimum of effective travel delays, which depend on the path flows $h$ :

$$
\begin{gathered}
v_{p}(h)=\operatorname{essinf}_{t \in\left[t_{0}, t_{f}\right]}\left\{\Psi_{p}(t, h)\right\}>0 \quad \forall p \in \mathcal{P} \\
v_{i j}(h)=\min _{p \in \mathcal{P}_{i j}}\left\{v_{p}(h)\right\} \quad \forall(i, j) \in \mathcal{W}
\end{gathered}
$$

where we use $p \in \mathcal{P}_{i j}$ to refer to a specific path contained in the set of paths $\mathcal{P}_{i j}$ connecting origin-destination (OD) pair $(i, j) \in \mathcal{W}$, and $\mathcal{W}$ is the set of all OD pairs. 


\section{Statement of the Dynamic User Equilibrium Problem}

As a consequence of notations introduced in Section 4, the dynamic user equilibrium model for finding path departure rates $h^{*} \geq 0$ is the following: ${ }^{2}$

$$
\left.\begin{array}{c}
h_{p}^{*}(t)>0, p \in \mathcal{P}_{i j} \Longrightarrow \\
\Psi_{p}^{*}\left(t, h^{*}\right)=v_{i j}\left(h^{*}\right) \quad \forall(i, j) \in \mathcal{W}
\end{array}\right\} \operatorname{DUE}(h, \Psi, \Lambda)
$$

where the set of admissible path flows

$$
\Lambda \equiv\left\{h \geq 0: \sum_{p \in \mathcal{P}_{i j}} \int_{t_{0}}^{t_{f}} h_{p}(t) d t=Q_{i j} \quad \forall(i, j) \in \mathcal{W}\right\}
$$

Furthermore, the effective path delay $\Psi\left(\cdot, h^{*}\right)$ is a solution of the dynamic network loading model one elects to describe a network of interest. Moreover, $Q=\left\{Q_{i j}:(i, j) \in \mathcal{W}\right\}$ is an exogenous, fixed trip table (matrix); and integration is in the sense of Lebesgue. Definition (5.8) provides the continuous time DUE problem we want to solve.

Ran et al. (1993); Ran and Boyce (1996); Ran et al. (1993) and Ran et al. (1996) also worked in continuous time and created a family of DUE models sharing a common foundation based on an equivalent optimal control formulation, for which they show the best responses of generic travelers constitutes a DUE flow pattern. Instead of viewing unit effective travel delay as an operator, they embedded the notion of network loading within their DUE model. They relied on highly specific arc entrance and exit flow functions, as well as novel flow propagation constraints that are quite different than the continuous time physical identities employed by Friesz et al. (2001), Kachani and Perakis (2009) and Perakis and Roels (2006).

Zhu and Marcotte (2000) observed a variational inequality for DUE may be stated when a DNL model is available. However, they were not able to directly prove the existence of solutions to (5.8); so they modified the model by using the following alternative flow conservation constraints

$$
\sum_{p \in \mathcal{P}_{i j}} h_{p}^{*}(t)=Q_{i j}(t) \quad \forall_{v}\left(t \in\left[t_{0}, t_{f}\right]\right) \quad \forall(i, j) \in \mathcal{W}
$$

in lieu of

$$
\sum_{p \in \mathcal{P}_{i j}} \int_{t_{0}}^{t_{f}} h_{p}^{*}(t) d t=Q_{i j} \quad \forall(i, j) \in \mathcal{W}
$$

In fact Zhu and Marcotte (2000) declare $Q_{i j}(t)$ is the O-D departure rate which is exogenously given. In (5.10), each $Q_{i j}(t)$ must be a flow (in vehicle per unit time), not a volume, to assure dimensional consistency. On the other hand, the representation (5.11) enjoys behavioral richness by allowing shift in departure time; in particular, (5.11) allows drivers to depart in platoons as is generally observed in real networks; (5.10) eliminates platoon formation. Friesz and Meimand (2014) and Han et al. (2015a) extend the formulation (5.8) to treat elastic travel demand, while Han et al (2015a) provide a comprehensive existence theory. Moreover, working in continuous time, Bliemer and Bovy (2003) have extended the Friesz et al. (1993) formulation by introducing multiple user classes, thereby creating a quasi-variational inequality.

Friesz et al. (1993) use measure-theoretic argument and show that the DUE problem (5.8) with simultaneous route and departure time choices can be reformulated as the following variational inequality:

$$
\text { find } h^{*} \in \Lambda \text { such that } \int_{t_{0}}^{t_{f}} \Psi_{p}\left(t, h^{*}\right)\left(h(t)-h^{*}(t)\right) d t \geq 0 \quad \forall h \in \Lambda
$$

A more recognizable form of the VI (5.12) is

$$
\text { find } h^{*} \in \Lambda \text { such that }\left\langle\Psi\left(h^{*}\right), h-h^{*}\right\rangle \geq 0 \quad \forall h \in \Lambda
$$

\footnotetext{
${ }^{2}$ Note that, by the measure-theoretic nature of the definition, (5.8) only needs to hold for almost every $t$.
} 
by invoking the inner product $\langle\cdot, \cdot\rangle$ in the Hilbert space $\left(L^{2}\left[t_{0}, t_{f}\right]\right)^{|\mathcal{P}|}$; see Appendix A.1 for more details.

We may show (5.8) is a a DVI for SRDT DUE rather easily by restating (5.9) as a differential equation and employing some elementary facts from the theory of optimal control. To that end, let us continue to treat the trip table $\left(Q_{i j}:(i, j) \in \mathcal{W}\right)$ as a fixed matrix of positive travel demands. We introduce a new state vector $y(t)=$ $\left(y_{i j}(t):(i, j) \in \mathcal{W}\right)$, which is readily recognized as cumulative departures; this vector, for any given instant of time $t$, belongs to $\mathbb{R}_{+}^{|W|}$. As a consequence the constraints (5.11) may be replaced with the following two-point boundary value problem:

$$
\frac{d y_{i j}(t)}{d t}=\sum_{p \in \mathcal{P}_{i j}} h_{p}^{*}(t), \quad y_{i j}\left(t_{0}\right)=0, \quad y_{i j}\left(t_{f}\right)=Q_{i j} \quad \forall(i, j) \in \mathcal{W}
$$

It is rather easy to see that expression

$$
h_{p}^{*}(t)>0, p \in \mathcal{P}_{i j} \Longrightarrow \Psi_{p}\left(t, h^{*}\right)=v_{i j} \quad \forall(i, j) \in \mathcal{W},
$$

from the definition $D U E(h, \Psi, \Lambda)$ given in (5.8), leads to the following chain of results for any given $(i, j) \in W$ :

$$
\begin{aligned}
\Psi_{p}\left(t, h^{*}\right) & \geq v_{i j} \quad \forall t, \forall p \in \mathcal{P}_{i j} \\
\Psi_{p}\left(t, h^{*}\right)\left(h_{p}(t)-h_{p}^{*}(t)\right) & \geq v_{i j}\left(h_{p}(t)-h_{p}^{*}(t)\right) \quad \forall t, \forall p \in \mathcal{P}_{i j} \\
\sum_{p \in \mathcal{P}_{i j}} \int_{t_{0}}^{t_{f}} \Psi_{p}\left(t, h^{*}\right)\left(h_{p}(t)-h_{p}^{*}(t)\right) d t & \geq v_{i j} \sum_{p \in \mathcal{P}_{i j}} \int_{t_{0}}^{t_{f}}\left(h_{p}(t)-h_{p}^{*}(t)\right) d t \\
& =v_{i j}\left(Q_{i j}-Q_{i j}\right)=0
\end{aligned}
$$

It is immediate that the problem $D U E(h, \Psi, \Lambda)$ may be expressed as: find $h^{*} \in \Lambda_{0}$ such that

$$
\sum_{p \in \mathcal{P}} \int_{t_{0}}^{t_{f}} \Psi_{p}\left(t, h^{*}\right)\left(h_{p}(t)-h_{p}^{*}(t)\right) d t \geq 0 \quad \forall h \in \Lambda_{0}
$$

where

$$
\Lambda_{0} \equiv\left\{h \geq 0: \frac{d y_{i j}(t)}{d t}=\sum_{p \in \mathcal{P}_{i j}} h_{p}^{*}(t), \quad y_{i j}\left(t_{0}\right)=0, \quad y_{i j}\left(t_{f}\right)=Q_{i j} \quad \forall(i, j) \in \mathcal{W}\right\}
$$

Problem (5.16) is a differential variational inequality as that notion is defined in Friesz and Mookherjee (2006); Friesz (2010); Friesz et al. (2011, 2013); Friesz and Meimand (2014); Han et al. (2015a) and Han et al. (2015b). We need to know some of the basic theory of differential variational inequalities before we turn to the question of how to compute solutions to (5.16); that background is given below in Appendix A.1, Appendix A.2 and Appendix A.4.

\section{Verifying the DVI is a DUE}

The existence of $h^{*}$ that satisfies (5.8) has been studied in Zhu and Marcotte (2000) and Han et al. (2013c), both based on a version of the Browder's fixed-point theorem in topological vector spaces (Browder, 1968). This section is dedicated to establishing the connection of DVI to the DUE model. In particular, by observation, the DVI (5.16) may be restated as the following optimal control problem:

$$
\min J=\sum_{(i, j) \in \mathcal{W}} v_{i j}\left(Q_{i j}-y_{i j}\left(t_{f}\right)\right)+\sum_{(i, j) \in \mathcal{W}} \sum_{p \in \mathcal{P}_{i j}} \int_{t_{0}}^{t_{f}} \Psi_{p}\left(t, h^{*}\right) h_{p}(t) d t \quad \forall h \in \Lambda_{0}
$$

However, the criterion $J$ depends on the solution $h^{*} \in \Lambda_{0}$, so it is futile to use (6.18) for calculations. The necessary conditions for optimal control (see Appendix A.4) may be employed to study the solutions of (6.18). In particular, the transversality conditions for each $(i, j) \in \mathcal{W}$ give us

$$
\lambda_{i j}\left(t_{f}\right)=\frac{\partial}{\partial y_{i j}\left(t_{f}\right)} \sum_{(i, j) \in \mathcal{W}} v_{i j}\left(Q_{i j}-y_{i j}\left(t_{f}\right)\right)=-v_{i j}=\mathrm{constant}
$$


The Hamiltonian of (6.18) is

$$
H=\sum_{(i, j) \in \mathcal{W}} \sum_{p \in \mathcal{P}_{i j}}\left(\Psi_{p}\left(t, h^{*}\right) h_{p}(t)+\lambda_{i j} \sum_{p \in \mathcal{P}_{i j}} h_{p}^{*}(t)\right)
$$

Hamiltonian is meant to be minimized with respect to its controls. Therefore the equilibrium path flows obey

$$
\min H \quad \text { s.t. } \quad-h \leq 0
$$

Since $\lambda_{i j}\left(t_{f}\right)=v_{i j}$, the Kuhn-Tucker conditions for $(i, j) \in \mathcal{W}$ and $p \in \mathcal{P}_{i j}$

$$
\begin{aligned}
\Psi_{p}\left(t, h^{*}\right)-v_{i j} & =\rho_{p} \geq 0 \\
\rho_{p} h_{p} & =0
\end{aligned}
$$

Thus

$$
\begin{array}{ccccc}
\text { (1) } \quad h_{p}^{*}(t)>0, p \in \mathcal{P}_{i j} & \Longrightarrow & \Psi_{p}\left(t, h^{*}\right)=v_{i j} & \text { DUE definition } \\
\text { (2) } \quad \Psi_{p}\left(t, h^{*}\right)>v_{i j}, p \in \mathcal{P}_{i j} & \Longrightarrow & h_{p}^{*}(t)=0 & \text { trivial flow condition }
\end{array}
$$

Expression (6.20) is, of course, what we mean by a dynamic user equilibrium.

\section{Fixed Point Formulation}

It is well known that a differential variational inequality may be represented as fixed-point problem whose subproblems are linear-quadratic optimal control problems (Friesz, 2010). In fact under mild regularity conditions, the differential variational inequality (5.16)-(5.17) is equivalent to this fixed-point problem:

$$
h=P_{\Lambda_{0}}\left[h-\alpha \Psi_{p}(t, h)\right],
$$

where $P_{\Lambda_{0}}[\cdot]$ is the minimum norm projection operator onto the convex and closed subset $\Lambda_{0}$, and $\alpha$ is a positive scalar. Consequently, we write

$$
h=\underset{z \in \Lambda_{0}}{\operatorname{argmin}} \frac{1}{2}\|h-\alpha \Psi(h)-z\|^{2}
$$

Assuming a constraint qualification holds, the Kuhn-Tucker conditions tell us that

$$
h_{p}-\alpha \Psi_{p}(t, h)-z_{p}=-v_{i j}^{0}-\rho_{p}^{0} \quad \forall(i, j) \in \mathcal{W}, p \in \mathcal{P}_{i j}
$$

for dual variables $v_{i j}^{0}$ and $\rho_{p}^{0}$ associated with the flow conservation and nonnegativity constraints, respectively. When we recognize that $z_{p}=h_{p}$, expression (7.22) yields

$$
\alpha \Psi_{p}(t, h)-v_{i j}^{0}=\rho_{p}^{0} \geq 0 \quad \forall(i, j) \in \mathcal{W}, p \in \mathcal{P}_{i j}
$$

The constant $\alpha>0$ may be absorbed by the dual variables; that is

$$
v_{i j}=\frac{v_{i j}^{0}}{\alpha} \text { and } \rho_{p}=\frac{\rho_{p}^{0}}{\alpha}
$$

so that

$$
\Psi_{p}(t, h)-v_{i j}=\rho_{p} \geq 0 \quad \forall(i, j) \in \mathcal{W}, p \in \mathcal{P}_{i j}
$$

In light of the complementary slackness conditions

$$
\begin{aligned}
\rho_{p} h_{p}=0 \quad \forall(i, j) \in \mathcal{W}, p \in \mathcal{P}_{i j} \\
\rho_{p} \geq 0 \quad \forall(i, j) \in \mathcal{W}, p \in \mathcal{P}_{i j}
\end{aligned}
$$


it is easy to see that (7.24) requires (6.20) to hold. Equivalence of the relevant DVI to the the fixed-point problem (7.21) is assured by convexity.

We turn now to the statement of a fixed-point algorithm made possible by (7.21). In particular we have the following:

\section{DUE Fixed-Point Algorithm}

Step 0. Initialization. Set $k=0$ and select an initial solution $h^{0} \in \Lambda_{0}$. Also select a suitable fixed step size $\alpha>0$ used in all iterations. ${ }^{3}$

Step 1. Dynamic Network Loading and Updating. Compute $\Psi\left(t, h^{k}\right)$ using a dynamic network loading model when flow is $h^{k} \in \Lambda_{0}$. For every origin-destination pair $(i, j) \in \mathcal{W}$, solve the following algebraic equation for $v_{i j}$ (where $[x]_{+} \doteq \max \{0, x\}$ assures non-negativity):

$$
\sum_{p \in \mathcal{P}_{i j}} \int_{t_{0}}^{t_{f}}\left[h_{p}^{k}(t)-\alpha \Psi_{p}\left(t, h^{k}\right)+v_{i j}\right]_{+} d t=Q_{i j}
$$

where $\alpha$ is a positive constant and $[\cdot]_{+}$is the projection onto the nonnegative orthant. Compute

$$
h^{k+1}(t)=h^{k}(t)-\alpha \Psi\left(t, h^{k}\right)+v
$$

where

$$
v=\left(v_{i j}:(i, j) \in \mathcal{W}\right)
$$

Step 2. Stopping Test. For a predetermined tolerance $\xi \in \mathbb{R}_{++}^{1}$, if

$$
\left\|h^{k+1}-h^{k}\right\| \leq \xi
$$

stop and declare $h^{k+1}$ a DUE solution. Otherwise set $k=k+1$ and go to Step 1 .

This algorithm has an associated proof of convergence provided the effective path delay operators are strongly monotone increasing. The convergence proof is easy to present in an informal way. First we note that the effective path delay operator is said to be be strongly monotone increasing when

$$
\left\langle\Psi^{k+1}-\Psi^{k}, h^{k+1}-h^{k}\right\rangle \geq K_{1}\left\|h^{k+1}-h^{k}\right\|^{2},
$$

where $K_{1}>0$ and the obvious notation $\Psi^{k} \doteq \Psi\left(\cdot, h^{k}\right)$ is employed. It will be convenient to restate (7.28) as

$$
-\left\langle\Psi^{k+1}-\Psi^{k}, h^{k+1}-h^{k}\right\rangle \leq-K_{1}\left\|h^{k+1}-h^{k}\right\|^{2}
$$

If we additionally assume Lipschitz continuity, we know

$$
\left\|\Psi^{k+1}-\Psi^{k}\right\|^{2} \leq K_{0}\left\|h^{k+1}-h^{k}\right\|^{2},
$$

where $K_{0} \in \mathbb{R}_{++}$. It is well known that the minimum norm projection operator is non-expansive in Hilbert space; therefore we examine

$$
\begin{aligned}
&\left\|h^{k+2}-h^{k+1}\right\|^{2} \leq\left\|\left(h^{k+1}-\alpha \Psi^{k+1}\right)-\left(h^{k}-\alpha \Psi^{k}\right)\right\|^{2} \\
&=\left\|\left(h^{k+1}-h^{k}\right)-\alpha\left(\Psi^{k+1}-\Psi^{k}\right)\right\|^{2} \\
&=\left\|h^{k+1}-h^{k}\right\|^{2}-2 \alpha\left\langle\Psi^{k+1}-\Psi^{k}, h^{k+1}-h^{k}\right\rangle+\alpha^{2}\left\|\Psi^{k+1}-\Psi^{k}\right\|^{2} \\
& \leq\left(1-2 \alpha K_{1}+\alpha^{2} K_{0}\right)\left\|h^{k+1}-h^{k}\right\|^{2} \\
& 8
\end{aligned}
$$


Thus, upon taking advantage of (7.29) and (7.30), we see that if

$$
\left(1-2 \alpha K_{1}+\alpha^{2} K_{0}\right)<1
$$

we have convergence. Expression (7.31) may be restated as

$$
K_{1}>\frac{\alpha K_{0}}{2}
$$

A formal proof of convergence of the fixed-point algorithm when applied to DUE DVIs with strongly monotone increasing effective delay operators is found in Friesz et al. (2011). The above analysis not withstanding, examples of effective delay operators that are not monotonically increasing have been reported. Therefore, at present, convergence of the fixed-point algorithm, as well as other algorithms, cannot be assured.

Remark 7.1. Intuitively from (7.27), and also clear from Sections 7.1 and 7.2, that the parameter $\alpha$ indicates the sensitivity of how the path flow vector $h^{k+1}$ depends on the travel cost $\Psi^{k}$ of the previous iteration. The convergence condition (7.32) suggests that small values of $\alpha$ are required to ensure convergence, provided the monotonicity property of the effective delay operator.

In the rest of this section, the fixed-point algorithm will be explicitly instantiated for some simple yet representative network examples, with closed expressions in continuous time. In order to highlight the updating part of the algorithm, we focus on scenarios where the dynamic network loading yields relatively simple forms of the effective path delays.

\subsection{Single Bottleneck Example}

We consider a network consisting of a single bottleneck, based on the Vickrey model (Vickrey, 1969; Han et al., $2013 \mathrm{a}, \mathrm{b})$. The capacity of the bottleneck is assumed to be $2000 \mathrm{veh} / \mathrm{hr}$, and the free-flow travel time is set to be zero for simplicity. Since our DUE problem involves departure time choice, we employ the following effective delay operator:

$$
\Psi(t, h)=\omega D(t, h)+ \begin{cases}\beta\left(T_{A}-A(t)\right) & A(t) \leq T_{A} \\ \gamma\left(A(t)-T_{A}\right) & A(t)>T_{A}\end{cases}
$$

where $\beta<\omega<\gamma$ are positive constants, $T_{A}$ is the desired time of arrival, and $A(t)=t+D(t, h)$ represents the arrival time. In the following illustrative calculation, we consider the time horizon of $[0,5]$ (in hr), and select these parameter values:

$$
\omega=0.8, \quad \beta=0.6, \quad \gamma=1.2, \quad T_{A}=3, \quad \alpha=200,
$$

where $\alpha$ is the positive constant appearing in the fixed-point algorithm; see Section 7.

Step 0. Initialization. We consider an initial path flow for the fixed-point algorithm:

$$
h^{0}(t)= \begin{cases}0, & t \in[0,2) \cup[4,5] \\ 1000, & t \in[2,4)\end{cases}
$$

Note that in the case of a single bottleneck, the path choice becomes irrelevant, and the DUE problem reduces to a departure-time-choice problem. In the following, we work out each intermediate iteration of the fixed-point algorithm, and interpret their means using continuous-time calculations.

ITERATION $k=0$. 
Step 1a. Dynamic Network Loading. Since the departure rate $h^{0}(t)$ is uniformly below the capacity of the bottleneck, according to the Vickrey model the bottleneck delay is zero, i.e. $D\left(t, h^{0}\right) \equiv 0$. Thus the effective delay reduces to:

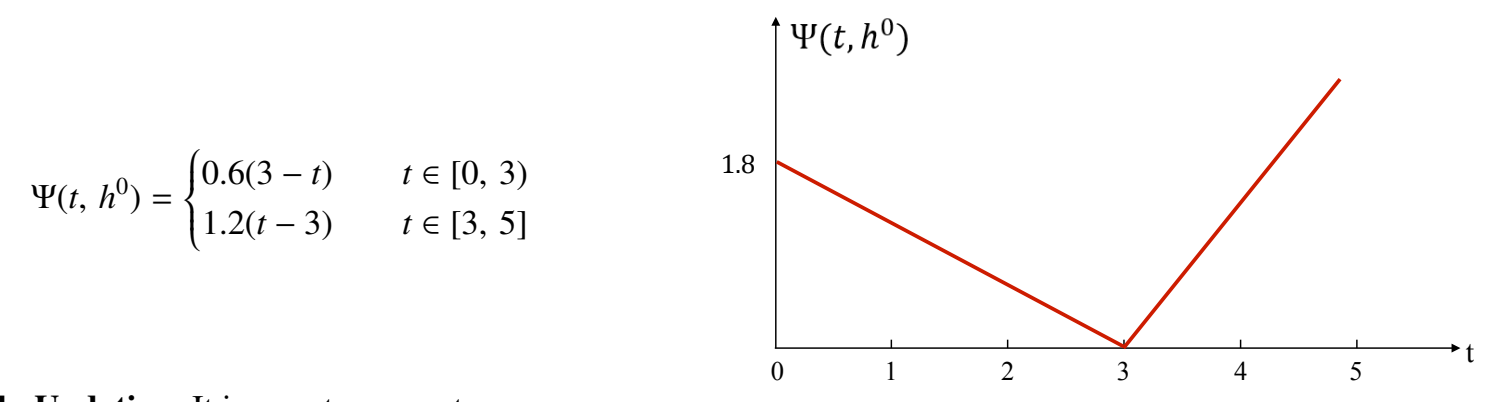

Step 1b. Updating. It is easy to compute:

$$
h^{0}(t)-\alpha \Psi\left(t, h^{0}\right)= \begin{cases}120 t-360 & t \in[0,2) \\ 120 t+640 & t \in[2,3) \\ -240 t+1720 & t \in[3,4) \\ -240 t+720 & t \in[4,5]\end{cases}
$$

The key step of the fixed-point update is the following equation:

$$
\int_{0}^{5}\left[h^{0}(t)-\alpha \Psi\left(t, h^{0}\right)+v_{i j}\right]_{+} d t=Q_{i j}=2000
$$

We need to find the unique value for $v_{i j}$ in order for (7.35) to hold. Given the explicit form (7.34), we readily derive that $v_{i j}=90$; thus the departure rate for $k=1$, as illustrated in Figure 1, is

$$
\begin{aligned}
h^{1}(t) & =\left[h^{0}(t)-\alpha \Psi\left(t, h^{0}\right)+v_{i j}\right]_{+} \\
& = \begin{cases}0 & t \in[0,2) \\
120 t+730 & t \in[2,3) \\
-240 t+1810 & t \in[3,4) \\
0 & t \in[4,5]\end{cases}
\end{aligned}
$$
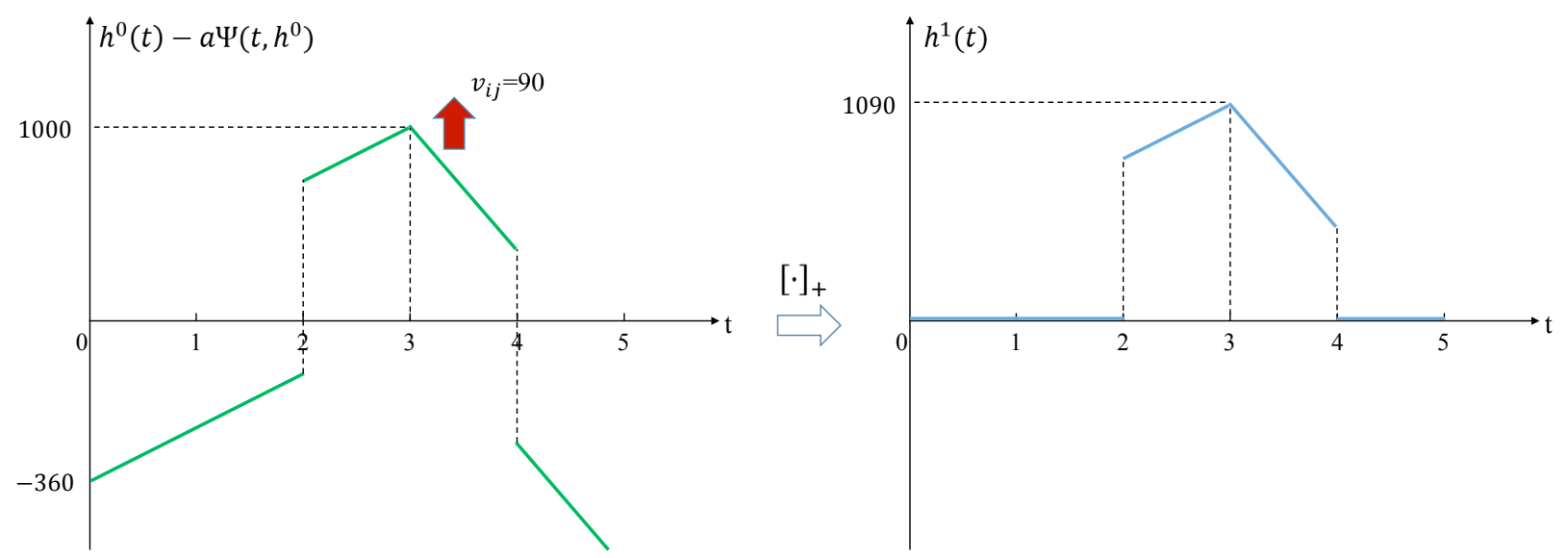

Figure 1: Determination of the dual variable $v_{i j}$ to yield $h^{1}(t)$.

ITERATION $k=1$. 
Step 1a. Dynamic Network Loading. Since the departure rate $h^{1}(t)$ is uniformly below the capacity of the bottleneck, the bottleneck delay is zero. Thus the effective delay is the same as (7.34).

Step 1b. Updating. From (7.36) and (7.34), we have that

$$
h^{1}(t)-\alpha \Psi\left(t, h^{1}\right)= \begin{cases}120 t-360 & t \in[0,2) \\ 240 t+370 & t \in[2,3) \\ -480 t+2530 & t \in[3,4) \\ -240 t+720 & t \in[4,5]\end{cases}
$$

Figure 2 illustrates how (7.37) is constructed. In order to satisfy the demand constraint, the dual variable is again calculated to be $v_{i j}=90$. Thus we have

$$
\begin{aligned}
h^{2}(t) & =\left[h^{1}(t)-\alpha \Psi\left(t, h^{1}\right)+v_{i j}\right]_{+} \\
& = \begin{cases}0 & t \in[0,2) \\
240 t+460 & t \in[2,3) \\
-480 t+2620 & t \in[3,4) \\
0 & t \in[4,5]\end{cases}
\end{aligned}
$$
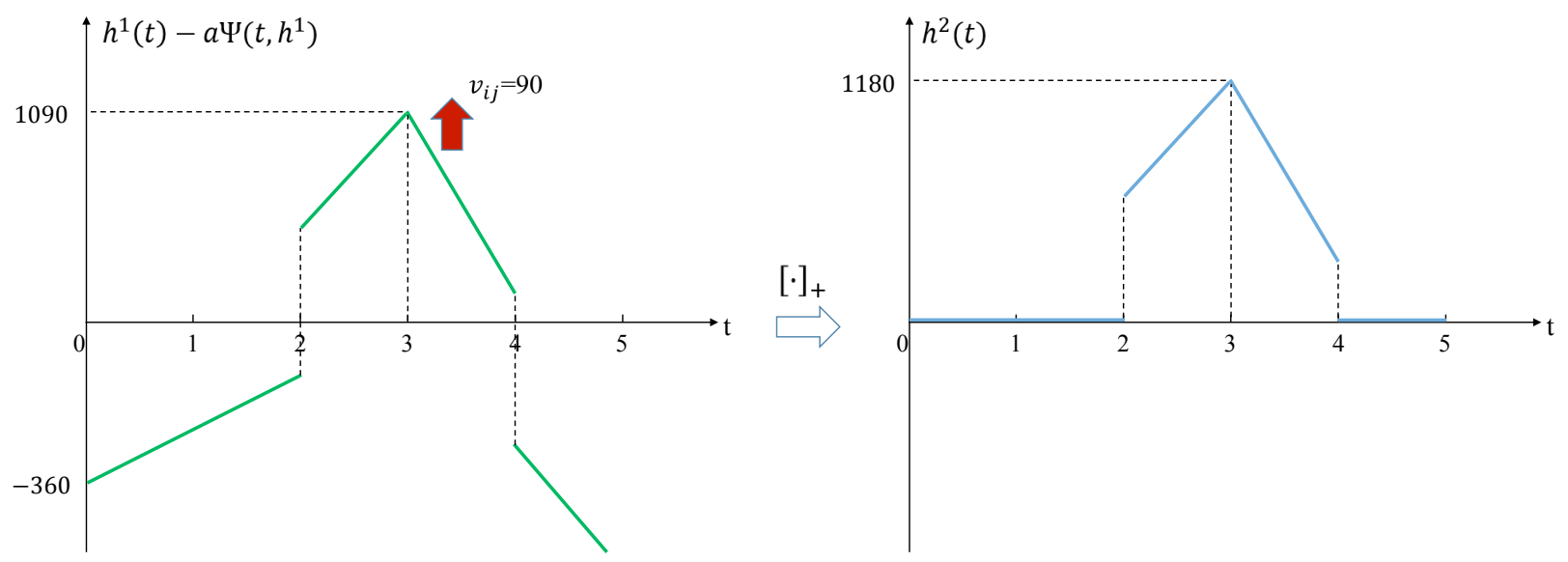

Figure 2: Determination of the dual variable $v_{i j}$ to yield $h^{2}(t)$.

Figure 3 summarizes the first three fixed-point iterations that produce $h^{0}(t), h^{1}(t)$ and $h^{2}(t)$. It can be seen that all the departures are concentrated in the interval $[2,4]$. In addition, as subsequent iterations are carried out, the algorithm tends to shift the departure mass to the time $t=3$ where the effective delay is the lowest. Moreover, because drivers departing during $[3,4]$ generally incur higher costs than those departing in $[2,3]$, more of them shift away from the departure window [3, 4].

\subsection{Three-Path Example}

In the second example, we consider the seven-arc, three-path network shown in Figure 4. The three paths are: $p_{1}=\left\{I_{1}, I_{2}, I_{5}, I_{7}\right\}, p_{2}=\left\{I_{1}, I_{2}, I_{4}, I_{6}, I_{7}\right\}, p_{3}=\left\{I_{1}, I_{3}, I_{6}, I_{7}\right\}$. The effective path delay structure is the same as (7.33), with the same values for $\omega, \beta$ and $\gamma$; now $\alpha=400$. In this example, the dynamic network loading is performed using the link transmission model (Yperman et al., 2005; Han et al., 2016), which captures vehicle spillback. The fixed travel demand between the O-D pair is $Q_{i j}=2000$. 


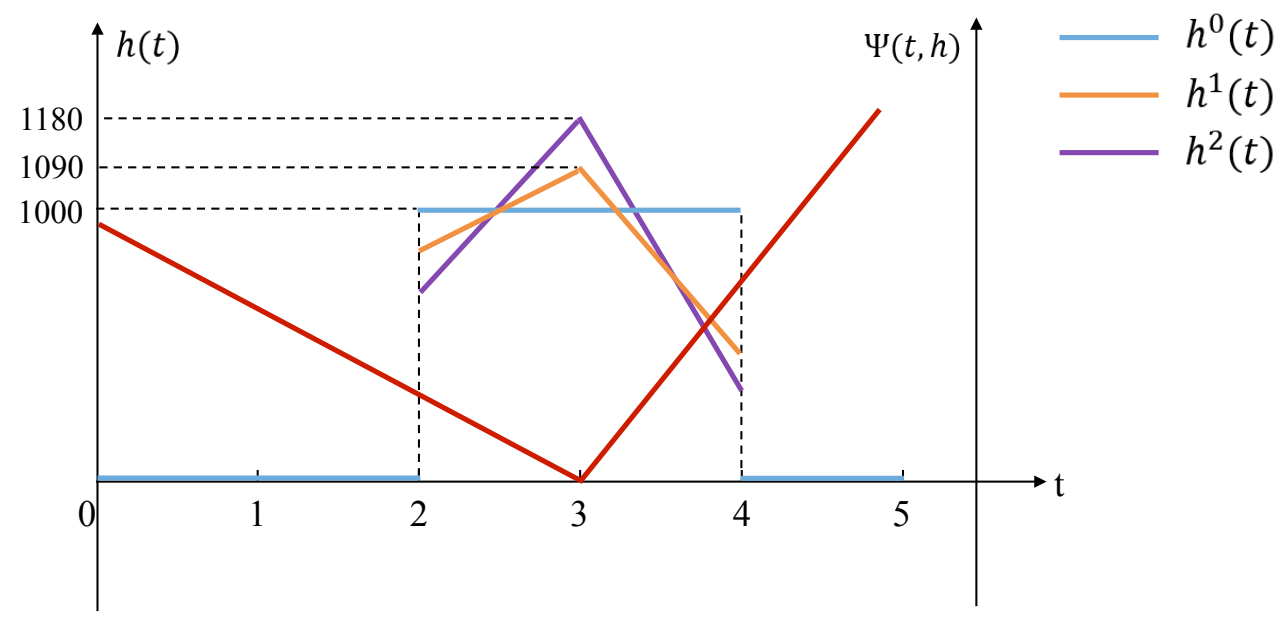

Figure 3: Summary of the first three iterates.

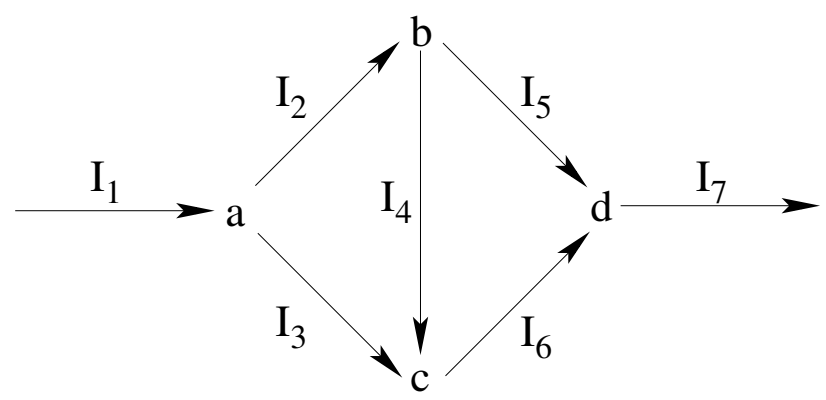

Figure 4: The seven-arc network.

Step 0. Initialization. The initial vector of path flows $h^{0}(t)$ is illustrated in Figure 5 and has these numerical values:

$$
h_{p_{1}}^{0}(t)=h_{p_{3}}^{0}(t) \equiv 0, \quad h_{p_{2}}^{0}(t)= \begin{cases}0 & t \in[0,1.5) \cup[3.5,5] \\ 1000 & t \in[1.5,3.5)\end{cases}
$$
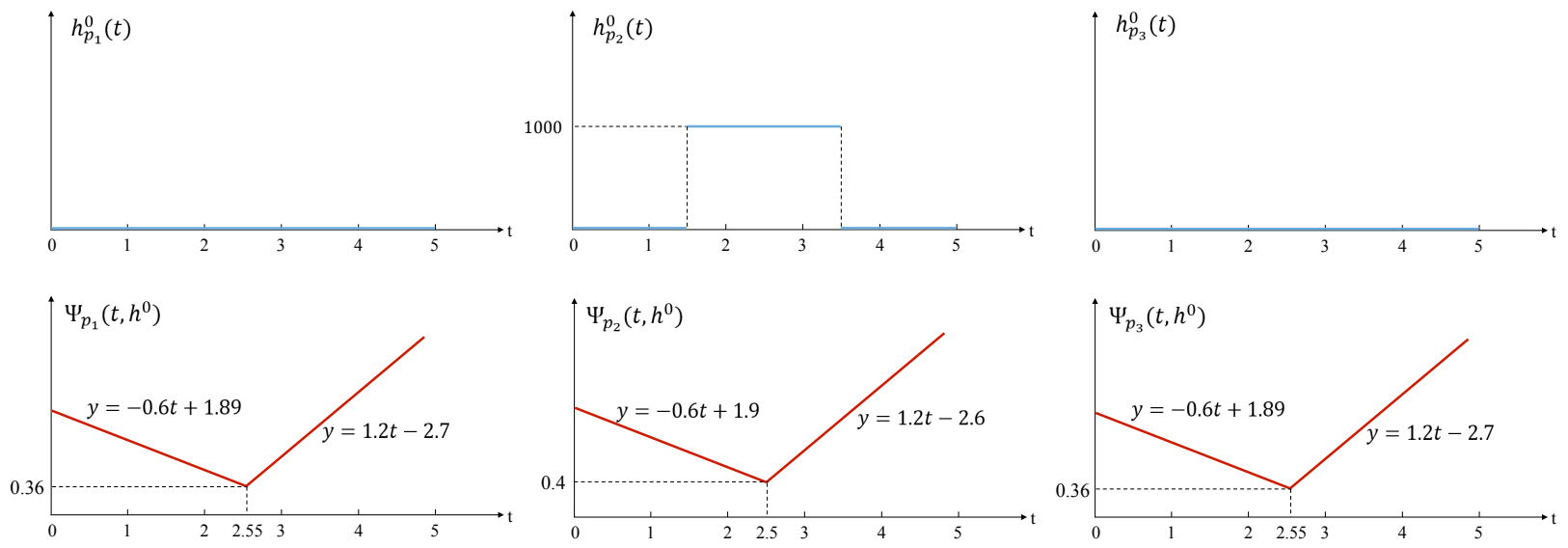

Figure 5: The initial path flows and their corresponding effective path delays. 
ITERATION $k=0$.

Step 1a. Dynamic Network Loading. The effective delays resulting from dynamic network loading are expressed in Figure 5 in closed form.

Step 1b. Updating. We readily calculate the following:

$$
\begin{array}{r}
h_{p_{1}}^{0}(t)-\alpha \Psi_{p_{1}}\left(t, h^{0}\right)=h_{p_{3}}^{0}(t)-\alpha \Psi_{p_{3}}\left(t, h^{0}\right)= \begin{cases}240 t-756 & t \in[0,2.55) \\
-480 t+1080 & t \in[2.55,5]\end{cases} \\
h_{p_{2}}^{0}(t)-\alpha \Psi_{p_{2}}\left(t, h^{0}\right)= \begin{cases}240 t-760 & t \in[0,1.5) \\
240 t+240 & t \in[1.5,2.5) \\
-480 t+2040 & t \in[2.5,3.5) \\
-480 t+1040 & t \in[3.5,5]\end{cases}
\end{array}
$$

Expressions (7.39)-(7.40) are illustrated in Figure 6.
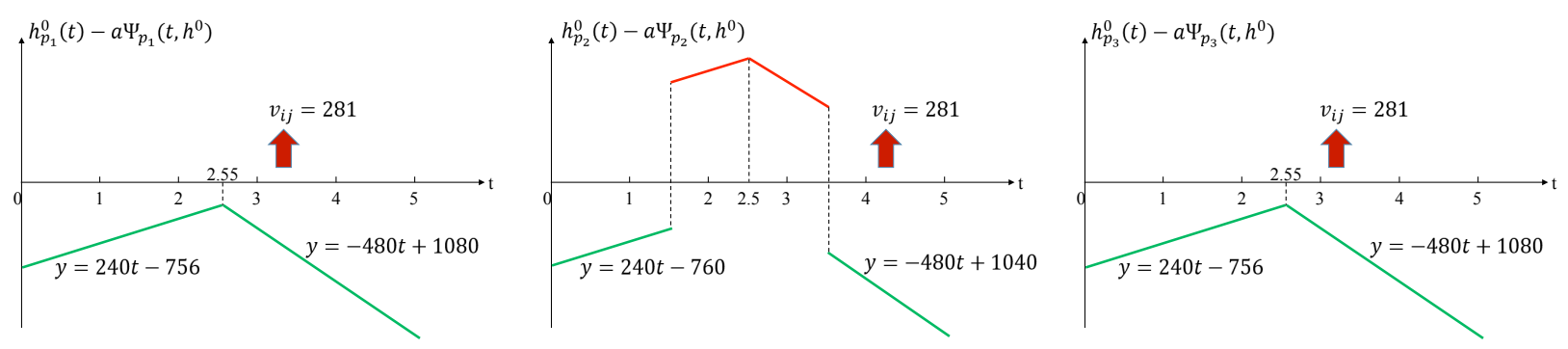

Figure 6: Determination of the dual variable $v_{i j}$.

The core of the fixed-point iteration is the determination of the dual variable $v_{i j}$. Note that the same $v_{i j}$ must be applied to all three quantities illustrated in Figure 6, so that the following holds:

$$
\int_{0}^{5}\left[h_{p_{1}}^{0}(t)-\alpha \Psi_{p_{1}}\left(t, h^{0}\right)+v_{i j}\right]_{+}+\left[h_{p_{2}}^{0}(t)-\alpha \Psi_{p_{2}}\left(t, h^{0}\right)+v_{i j}\right]_{+}+\left[h_{p_{3}}^{0}(t)-\alpha \Psi_{p_{3}}\left(t, h^{0}\right)+v_{i j}\right]_{+} d t=Q_{i j}
$$

Based on the closed-form expressions (7.39)- (7.40), we can calculate that $v_{i j}=281$. The resulting path flows for the next iteration are:

$$
\begin{gathered}
h_{p_{1}}^{1}(t)=h_{p_{3}}^{1}(t)= \begin{cases}0 & t \in[0,1.98) \\
240 t-475 & t \in[1.98,2.55) \\
-480 t+1361 & t \in[2.55,2.84) \\
0 & t \in(2.84,5]\end{cases} \\
h_{p_{2}}^{1}(t)= \begin{cases}0 & t \in[0,1.5) \\
240 t+521 & t \in[1.5,2.5) \\
-480 t+2321 & t \in[2.5,3.5) \\
0 & t \in[3.5,5]\end{cases}
\end{gathered}
$$

These path flows are illustrated in Figure 7.

ITERATION $k=1$.

Step 1a. Dynamic Network Loading. Due to the absence of significant congestion, the effective delays remain the same as those shown in 5. 

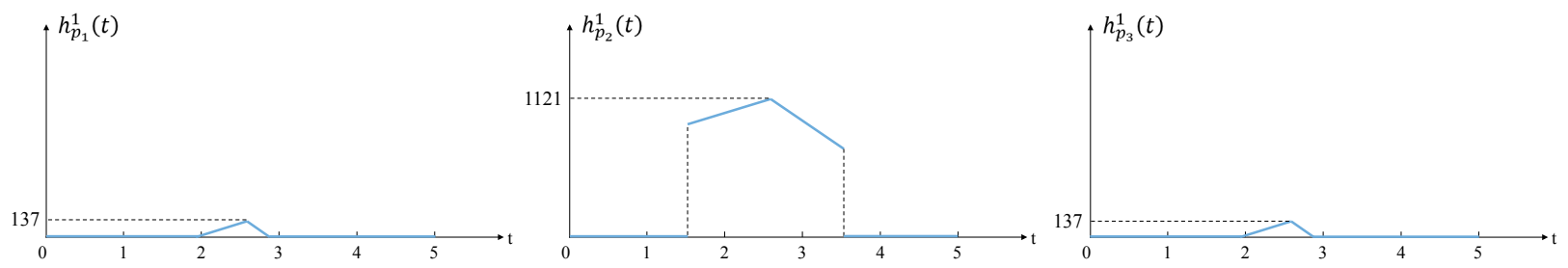

Figure 7: The path flows after the first fixed-point iteration.

Step 1b. Updating. Given the effective delays, we have that

$$
\begin{gathered}
h_{p_{1}}^{1}(t)-\alpha \Psi_{p_{1}}\left(t, h^{1}\right)=h_{p_{3}}^{1}(t)-\alpha \Psi_{p_{3}}\left(t, h^{1}\right)= \begin{cases}240 t-756 & t \in[0,1.98) \\
480 t-1231 & t \in[1.98,2.55) \\
-960 t+2441 & t \in[2.55,2.84) \\
-480 t+1080 & t \in[2.84,5]\end{cases} \\
h_{p_{2}}^{1}(t)-\alpha \Psi_{p_{2}}\left(t, h^{1}\right)= \begin{cases}240 t-760 & t \in[0,1.5) \\
480 t-239 & t \in[1.5,2.5) \\
-960 t+3361 & t \in[2.5,3.5) \\
-480 t+1040 & t \in[3.5,5]\end{cases}
\end{gathered}
$$
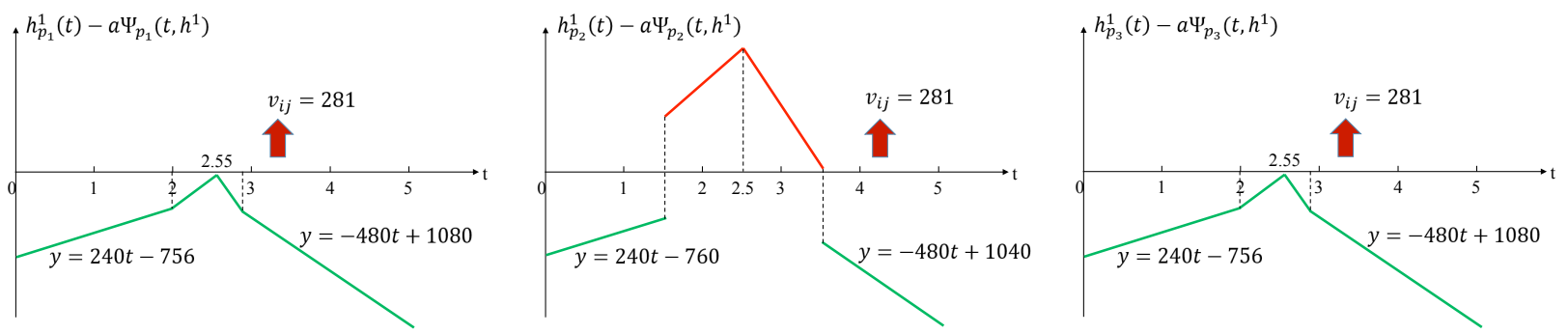

Figure 8: Determination of the dual variable $v_{i j}$.

See Figure 8 for an illustration of these quantities. Based on the closed-form expressions (7.41)- (7.42), we find the unique value $v_{i j}=281$. The resulting path flows from the second iteration are:

$$
\begin{gathered}
h_{p_{1}}^{1}(t)=h_{p_{3}}^{1}(t)= \begin{cases}0 & t \in[0,1.98) \\
480 t-950 & t \in[1.98,2.55) \\
-960 t+2722 & t \in[2.55,2.84) \\
0 & t \in(2.84,5]\end{cases} \\
h_{p_{2}}^{1}(t)= \begin{cases}0 & t \in[0,1.5) \\
480 t+42 & t \in[1.5,2.5) \\
-960 t+3642 & t \in[2.5,3.5) \\
0 & t \in[3.5,5]\end{cases}
\end{gathered}
$$

These path flows are illustrated in Figure 9

The dynamic network loading performed for fixed-point iterations indicate that the effective path delays along $p_{2}$ are in general higher than those along $p_{1}$ and $p_{3}$. As a result, in two consecutive iterations we observe the drivers moving toward alternatives with lower costs. In particular, at the end of the first iteration, not only do drivers travel 

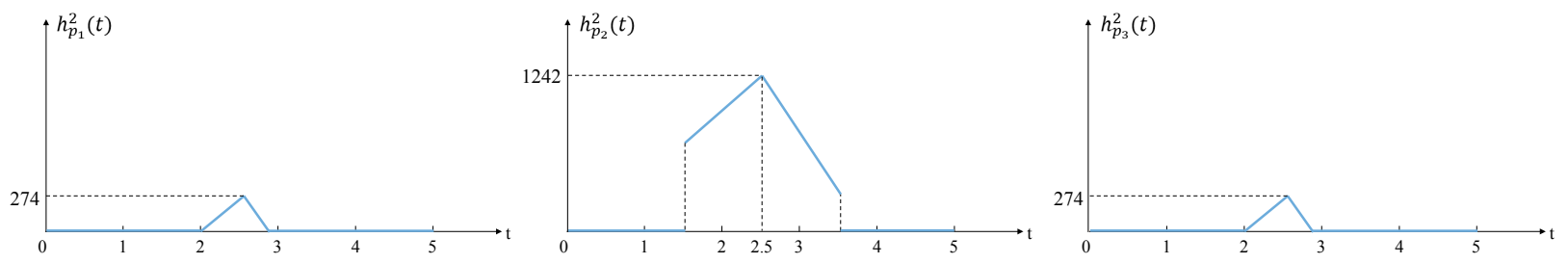

Figure 9: The path flows after the second fixed-point iteration.

on path $p_{2}$ move toward $t=2.5$ as their departure time, because the cost there is lower, part of them also move to paths $p_{1}$ and $p_{3}$ with lower costs (see Figure 7). At the end of the second iteration, such a shifting of choices is more pronounced (see Figure 9). As is intuitive, the fixed-point algorithm produces an evolutionary pattern that is reminiscent of day-to-day route choice models. However, note must be made that the algorithm presented here is by no means a behavioral model; rather, it is purely a consequence of the fixed-point formulation of DUE, a formulation based on a minimum norm projection subproblem that is a linear-quadratic optimal control problem.

\section{Concluding Remarks}

We have shown in our analysis the following:

1. Dynamic user equilibrium can be represented as a differential variational inequality (DVI).

2. DVI theory suggests a fixed-point algorithm for calculating DUE solutions.

3. The fixed-point algorithm makes the calculation of state variables (cumulative traffic volumes) a first order initial value problem wherein the right hand side of each differential equation depends only on time.

4. Similarly, the calculation of adjoint variables (dynamic dual variables for the state dynamics) is based on a first order terminal value problem wherein the right hand side of each differential equation depends only on time.

5. The state initial value problems and the adjoint final value problems, presented in the appendix, are decoupled. Thus, one avoids the need to directly solve a two-point boundary value problem, which is one of the most challenging aspects of optimal control and DVI problems.

It is abundantly clear that the DVI arises from a VI, where the former provides a variational problem that is natural generalization of an optimal control problem to a Nash-like game. The consequences of this include but are not limited to the following:

(i) The optimal-control-like aspect of DVIs is important because it allows the large literature on differential games to be exploited to find numerical schemes for computing DUE.

(ii) The DVI allows immediate affirmation that the dual variables for terminal-time constraints are identical to the adjoint variables in magnitude, and both are constant in time.

(iii) The minimum effective travel delay is the dual variable, for each OD pair.

(iv) In light of (i), (ii) and (iii), the DUE nature of DVI solutions is confirmed by inspection.

(v) Each of the aforementioned dual variables may be found by a one-dimensional line search.

(vi) The fact that all nonzero departure rates are singular solutions becomes obvious and means the departure rates are piecewise smooth.

When the features listed immediately above, whether used in continuous or discrete time, provide a simple, powerful means of solving the DUE problem as a fixed-point problem. The fixed-point algorithm needs minor modifications as explained in Friesz et al. (2011), Han et al. (2015a) and Han et al. (2015b) to enhance its performance and prove convergence for strongly monotone operators. Since the path delay operators encountered in DUE/DNL modeling are 
known to be nonmonotone, the fixed-point algorithm is a heuristic in practice. However, that is presently the case with all DUE/DNL algorithms proposed to date. Mastery of a substantial fraction of the body of thought pertaining to DVI formulation and fixed-point solution of DUE problems can be obtained using the results provided in this paper. Other elementary algorithms, especially gap function methods, may be similarly motivated. Numerical tests reported in Han et al. (2015a) and Han et al. (2015b) provide clear evidence that fixed-point algorithms are quite effective on small and medium size networks. To date, we find no compelling evidence that more sophisticated algorithms are needed for deliberate transportation planning analyses.

\section{Appendix A. Infinite Dimensional Mathematical Programming and Optimal Control}

In this appendix, we briefly review background material that is employed in the main body of our paper when discussing differential variational optimal control.

\section{Appendix A.1. Gâteaux Derivatives, Gradients, and Kuhn-Tucker Conditions}

The derivative of a function $f(x)$ of a real variable $x \in \mathbb{R}^{1}$ is defined by

$$
\frac{d f}{d x}=\lim _{h \rightarrow 0} \frac{f(x+h)-f(x)}{h}
$$

provided the indicated limit exists. This definition is not readily generalized to form the derivative of a functional $J(v)$, which may be thought of as a function of a function $v$, which assumes scalar values. Consequently, in order to articulate necessary conditions for optimality in infinite-dimensional mathematical programming we rely on the notion of a directional derivative. In fact, we now introduce the notion of a Gâteaux derivative, or G-derivative, which we define as follows:

Definition Appendix A.1. (G-derivative). Let $V$ be a topological vector space and $J$ a functional defined on $V$. Provided it exists, the limit

$$
\lim _{\theta \rightarrow 0 \in \mathbb{R}^{1}} \frac{J(v+\theta \phi)-J(v)}{\theta} \equiv \delta J(v, \phi)
$$

is the Gâteaux derivative ( $G$-derivative) at $v \in V$ in the direction $\phi \in V$. If the limit $\delta J(v, \phi)$ exists for all $\phi \in V$, we say that the functional $J$ is differentiable in the sense of Gâteaux (G-differentiable) at $v \in V$.

The G-derivative is a generalization of the idea of a directional derivative familiar in finite-dimensional spaces to infinite-dimensional spaces. Here, the medium in which the notion of G-derivative is conceived is referred to as topological vector space, which is one of the basic structures investigated in functional analysis (Rudin, 2006). For instance, the Hilbert space of square integrable functions on $\left[t_{0}, t_{f}\right] \subset \mathbb{R}$, with the inner product defined as

$$
\langle f, g\rangle \doteq \int_{t_{0}}^{t_{f}} f(t) g(t) d t
$$

is a topological vector space. The same goes for the m-fold space $\left(L^{2}\left[t_{0}, t_{f}\right]\right)^{m}$ as we studied in Section 4:

$$
\langle f, g\rangle \doteq \int_{t_{0}}^{t_{f}}[f(t)]^{T} g(t) d t
$$

where the superscript $T$ indicates transpose of the vector-valued function.

Given the pedagogical nature of the paper, as well as the space limitation that does not allow complete and rigorous elaboration of a topological vector space and its properties, we simplify the presentation in the rest of the paper by restricting the topological vector space $V$ to be the space $\left(L^{2}\left[t_{0}, t_{f}\right]\right)^{m}$, with the inner product defined in (A.3).

When $J(v)$ is a functional over $V$ and if there exists $\nabla J(v) \in V$ such that

$$
\delta J(v, \phi)=\langle\nabla J(v), \phi\rangle \quad \forall \phi \in V,
$$


we say $\nabla J(v)$ is the gradient of $J$ at $v$. In (A.4) the inner production is associated with the underlying topological vector space $V$. Because of the informal nature of this paper we will assume that $\nabla J(v)$ exists and is well defined for any infinite dimensional mathematical program we encounter in this paper.

Now let us consider the following infinite dimensional mathematical program:

$$
\min J(v) \quad \text { s.t. } \quad v \in U \equiv\left\{v: g_{i}(v) \leq 0 \quad i \in[1, m]\right\} \subset V
$$

We will assume without further comment that some constraint qualification is in effect, $U$ is convex, $V$ is a real Hilbert space, and $J(v)$ is differentiable in the sense of Gâteaux. Furthermore, let the $g_{i}(v)$ for $i \in[1, m]$ be functionals which are also differentiable in the sense of Gâteaux, so that the G-derivatives of $J(v)$ and of the $g_{i}(v)$ are denoted by

$$
\delta J(v, \phi) \quad \text { and } \quad \delta g_{i}(v, \phi) \quad i \in[1, m]
$$

for directions $\phi \in V$. Additionally, we assume the gradients of $J(v)$ and $g_{i}(v)$, for $i \in[1, m]$, may be expressed via these relationships:

$$
\begin{aligned}
& \delta J(v, \phi)=\langle\nabla J(v), \phi\rangle \\
& \delta g_{i}(v, \phi)=\left\langle\nabla g_{i}(v), \phi\right\rangle \quad i \in[1, m]
\end{aligned}
$$

With the apparatus introduced above and the previous invocation of some constraint qualification, it is well known [see Friesz (2010)] that there are valid Kuhn-Tucker necessary conditions for the infinite dimensional mathematical program (A.5), namely that there exist multipliers $\eta_{i}$ for all $i \in[1, m]$ such that, for $v^{*}$ a local minimum $J(v)$ on $U$, the following conditions hold:

$$
\begin{gathered}
\nabla J\left(v^{*}\right)+\sum_{i=1}^{m} \eta_{i} \nabla g_{i}\left(v^{*}\right)=0 \\
\eta_{i} g_{i}\left(v^{*}\right)=0 \quad i \in[1, m] \\
\eta_{i} \geq 0 \quad i \in[1, m]
\end{gathered}
$$

If in addition, $J(v)$ is convex on $U$ and the $g_{i}(v)$ are convex on $U$ for all $i \in[1, m]$, then conditions (A.9), (A.10), and (A.11) are also sufficient for $v^{*}$ to be a global minimum of $J(v)$ on $U$. While the Kuhn-Tucker conditions (A.9), (A.10), and (A.11) are not surprising, they will be fundamental to understanding how a computational scheme will be constructed for problems like (A.5).

Another key result, which can be proven rigorously [see Friesz (2010)], is that there is a variational equality necessary condition for (A.5).

Theorem Appendix A.2. (Variational inequality necessary condition) Take $J(v)$ to be a functional on $V$ that is $G$-differentiable at $v^{*} \in U$, and let $U \subset V$ be a convex set. A necessary condition for $v^{*} \in U$ to be a minimum of $J$ on $U$ is

$$
\delta J\left(v^{*}, v-v^{*}\right) \geq 0 \quad \forall v \in U
$$

\section{Appendix A.2. The Optimal Control Problem Defined}

A form of optimal control problem that is widely used in science, engineering and economics is this one:

$$
\min J(u)=K\left[x\left(t_{f}\right), t_{f}\right]+\int_{t_{0}}^{t_{f}} f_{0}(x, u, t) d t
$$

subject to

$$
\begin{aligned}
\frac{d x}{d t} & =f(x, u, t) \\
x\left(t_{0}\right) & =x_{0} \in \mathbb{R}^{n} \\
u & \in U \\
\Theta\left[x\left(t_{f}\right), t_{f}\right] & =0
\end{aligned}
$$


where $x(t) \in \mathbb{R}^{n}$ and $u(t) \in \mathbb{R}^{m}$ for each $t \in\left[t_{0}, t_{f}\right], t_{0}$ and $t_{f}$ are fixed scalars, $U$ is convex, and $x_{0}$ is fixed. Moreover, the functions $K, \Theta, f_{0}$, and $f$ will have whatever continuity and differentiability we require of them. In particular we take the control vector $u$ to have jump discontinuities. It is customary to restate (A.13)-(A.17) as

$$
\min J(u)=\Phi\left[x\left(t_{f}\right), t_{f}\right]+\int_{t_{0}}^{t_{f}} f_{0}(x, u, t) d t
$$

subject to

$$
\begin{aligned}
\frac{d x}{d t} & =f(x, u, t) \\
x\left(t_{0}\right) & =x_{0} \in \mathbb{R}^{n} \\
u & \in U
\end{aligned}
$$

where

$$
\Phi\left[x\left(t_{f}\right), t_{f}\right] \equiv K\left[x\left(t_{f}\right), t_{f}\right]+v^{T} \Theta\left[x\left(t_{f}\right), t_{f}\right]
$$

We will subsequently have need to refer to the state operator for the above optimal control problem, that operator is conveniently stated as

$$
x(u, t)=\arg \left\{\frac{d x}{d t}=f(x, u, t), x\left(t_{0}\right)=x_{0}\right\}
$$

For simplicity, we simply assume that the state operator exists and is well-defined. Traditionally the necessary conditions for this problem are expressed via the Hamiltonian:

$$
H(x, u, \lambda, t) \equiv f_{0}(x, u, t)+\lambda^{T} f((x, u, t)
$$

where $\lambda(t) \in \mathbb{R}^{n}$ for each $t \in\left[t_{0}, t_{f}\right]$ is the so-called vector of adjoint variables, which may be linked to dynamic dual variables.

\section{Appendix A.3. The Gradient of the Criterion Functional}

It is critical to know, in reference to the optimal control problem (A.13)-(A.17), that

$$
\nabla_{u} J(u)=\lambda^{T} \frac{\partial f}{\partial u}+\frac{\partial f_{0}}{\partial u}=\frac{\partial H}{\partial u}
$$

This result is a direct consequence of analyzing the optimal control problem (A.18)-(A.21) using the notion of a Gderivative; we provide an overview of how (A.24) is derived.. That exercise involves the notions presented in Section Appendix A.1, along with integration by parts, and some tedious algebra. In particular, note that the state dynamics may be expressed as an integral equation:

$$
x(u, t)=x\left(t_{0}\right)+\int_{t_{0}}^{t} f[x(u, \xi), u, \xi] d \xi
$$

It is immediate that

$$
x(u+\theta \rho, t)=x\left(t_{0}\right)+\int_{t_{0}}^{t} f[x(u+\theta \rho), u+\theta \rho, \xi] d \xi
$$

Consequently,

$$
\delta x(u, \rho ; t)=\int_{t_{0}}^{t}\left\{\frac{\partial f[x(u, \xi), u, \xi]}{\partial x} \delta x(u, \rho ; t)+\frac{\partial f[x(u, \xi), u, \xi]}{\partial u} \delta u(\rho)\right\} d \xi
$$

where of course the G-derivative of $u$ obeys

$$
\delta u(\rho)=\lim _{\theta \longrightarrow 0} \frac{(u+\theta \rho)-u}{\theta}=\rho
$$


Employing the shorthand $y=\delta x(u, \rho ; t)$, we may show that $y$ obeys

$$
\frac{d y}{d t}=\frac{\partial f}{\partial x} y+\frac{\partial f}{\partial u} \rho ; y\left(t_{0}\right)=0
$$

which is recognized as an initial value problem. The G-derivative of $J$ may be shown to obey

$$
\delta J(u, \rho)=\frac{\partial \Phi\left[x\left(t_{f}\right), t_{f}\right]}{\partial x} y\left(t_{f}\right)+\int_{t_{0}}^{t_{f}}\left[\frac{\partial f_{0}}{\partial x} y+\frac{\partial f_{0}}{\partial u} \rho\right] d t
$$

We introduce adjoint variables $\lambda$ defined by the following final value problem

$$
-\frac{d \lambda}{d t}=\left(\frac{\partial f}{\partial x}\right)^{T} \lambda+\left(\frac{\partial f_{0}}{\partial x}\right)^{T} ; \lambda\left(t_{f}\right)=\frac{\partial \Phi\left[x\left(t_{f}\right), t_{f}\right]}{\partial x\left(t_{f}\right)}
$$

so that

$$
\delta J(u, \rho)=\int_{t_{0}}^{t_{f}}\left[-\left(\frac{d \lambda}{d t}\right)^{T} y-\lambda^{T} \frac{\partial f}{\partial x} y+\frac{\partial f_{0}}{\partial u} \rho\right] d t
$$

Note that

$$
\left[\lambda^{T} y\right]_{t_{0}}^{t_{f}}=\left[\lambda\left(t_{f}\right)\right]^{T} y\left(t_{f}\right)-\left[\lambda\left(t_{0}\right)\right]^{T} y\left(t_{0}\right)=\frac{\partial \Phi\left[x\left(t_{f}\right), t_{f}\right]}{\partial x\left(t_{f}\right)} y\left(t_{f}\right)
$$

due to (A.26) and the fact that $y\left(t_{0}\right)=0$, integration by parts yields

$$
\begin{aligned}
\int_{t_{0}}^{t_{f}}-\left(\frac{d \lambda}{d t}\right)^{T} y d t & =\int_{t_{0}}^{t_{f}} \lambda^{T} \frac{d y}{d t} d t-\left[\lambda^{T} y\right]_{t_{0}}^{t_{f}} \\
& =\int_{t_{0}}^{t_{f}} \lambda^{T} \frac{d y}{d t} d t-\frac{\partial \Phi\left[x\left(t_{f}\right), t_{f}\right]}{\partial x} y\left(t_{f}\right) \\
& =\int_{t_{0}}^{t_{f}} \lambda^{T}\left[\frac{\partial f}{\partial x} \cdot y+\frac{\partial f}{\partial u} \cdot \rho\right] d t-\frac{\partial \Phi\left[x\left(t_{f}\right), t_{f}\right]}{\partial x} y\left(t_{f}\right)
\end{aligned}
$$

It follows that

$$
\delta J(u, \rho)=\int_{t_{0}}^{t_{f}}\left[\lambda^{T} \frac{\partial f}{\partial u}+\frac{\partial f_{0}}{\partial u}\right] \rho d t
$$

By virtue of the definition of the Hamiltonian (A.23), we have

$$
\delta J(u, \rho)=\int_{t_{0}}^{t_{f}}\left[\frac{\partial H}{\partial u} \rho\right] d t
$$

as an expression for the G-derivative of the criterion with respect to $u$. By Theorem Appendix A.2, optimality requires $u^{*} \in U$ to obey

$$
\delta J\left(u^{*}, \rho\right) \geq 0 \quad \forall \rho \geq 0
$$

By statement (A.30), we know

$$
\delta J\left(u^{*}, \rho\right)=\left\langle\nabla J\left(u^{*}\right),\left(u-u^{*}\right)\right\rangle \quad \forall u \in U
$$

Hence

$$
[\nabla J(u)]_{i}=\frac{\partial H}{\partial u_{i}}
$$

for $t \in\left[t_{0}, t_{f}\right]$, which is the component-wise version of (A.24). 


\section{Appendix A.4. Necessary Conditions for Optimal Control}

The analysis of Section Appendix A.2 also provides the necessary conditions for optimal control. In particular if $\left(x^{*}, u^{*}, \lambda^{*}, t\right)$ is an optimal solution of (A.18)-(A.21), then the following observations may be made:

1. From (A.12) and (A.31), we obtain the key necessary condition obeyed by an optimal solution $\left(x^{*}, u^{*}, \lambda^{*}\right)$ of (A.18)-(A.21), namely the following variational inequality:

$$
\sum_{i=1}^{m} \frac{\partial H\left(x^{*}, u^{*}, \lambda^{*}, t\right)}{\partial u_{i}}\left(u_{i}-u_{i}^{*}\right) \geq 0 \quad \forall u \in U \quad \forall t \in\left[t_{0}, t_{f}\right],
$$

which is widely known as the minimum principle since it is a necessary condition for minimizing the Hamiltonian with respect to the controls $u \in U$.

2. The statement of optimal control problem (A.18)-(A.21) contains the state initial value problem:

$$
\begin{aligned}
\frac{d x^{*}}{d t} & =f\left(x^{*}, u^{*}, t\right) \\
x^{*}\left(t_{0}\right) & =x_{0}
\end{aligned}
$$

3. Conditions (A.26) are the adjoint final value problem:

$$
\begin{aligned}
(-1) \frac{d \lambda^{*}}{d t} & =\nabla_{x} H^{*} \\
\lambda\left(t_{f}\right) & =\frac{\partial \Phi\left[x^{*}\left(t_{f}\right), t_{f}\right]}{\partial x\left(t_{f}\right)}
\end{aligned}
$$

Appendix A.5. Solving a DVI Using a Fixed-Point Representation

Under mild regularity conditions, it may be proven that a DVI of the form:

$$
\begin{aligned}
& \text { find } u^{*} \in U \text { such that } \\
& \qquad\left\langle F\left(x\left(u^{*}, t\right), u^{*}, t\right), u-u^{*}\right\rangle \geq 0 \quad \forall u \in U
\end{aligned}
$$

is equivalent to the following fixed-point problem:

$$
u^{*}=P_{U}\left[u^{*}-\rho F\left(x\left(u^{*}, t\right), u^{*}, t\right)\right]
$$

where $P_{U}$ [.] is the minimum norm projection onto $U, \rho>0$ is an arbitrary positive constant, and appropriate regularity conditions are satisfied. The most important regularity conditions are: the existence of the state operator; the strong continuity and G-differentiability of the state operator; the continuity of $F$; the continuous differentiability of $f$ and $\Theta$; and the convexity of $U$. The result may be proven by direct application of the minimum principle and associated optimality conditions to the minimum norm problem intrinsic to projection operator $P_{U}$ [.]. See Friesz (2010) for the details suppressed here.

\section{Appendix A.6. The Unembellished Fixed-Point Algorithm and Its Convergence}

Naturally there is an associated fixed-point algorithm based on the iterative scheme:

$$
u^{k+1}=P_{U}\left[u^{k}-\rho F\left(x\left(u^{k}, t\right), u^{k}, t\right)\right]
$$

The positive scalar $\rho$ may be chosen empirically to assist convergence and may even be changed as the algorithm progresses. The detailed structure of the fixed-point algorithm is given below:

\section{Fixed-Point Algorithm}


Step 0. Initialization. Identify an initial feasible solution $u^{0} \in U$ and set $k=0$.

Step 1. Solve the optimal control subproblem. Solve the following optimal control subproblem:

$$
\min _{v} J^{k}(v)=\gamma^{T} \Theta\left[x\left(t_{f}\right), t_{f}\right]+\int_{t_{0}}^{t_{f}} \frac{1}{2}\left[u^{k}-\rho F\left(x^{k}, u^{k}, t\right)-v\right]^{2} d t
$$

subject to

$$
\begin{aligned}
\frac{d x}{d t} & =f(x, v, t) \\
x\left(t_{0}\right) & =x_{0} \\
v & \in U
\end{aligned}
$$

Call the solution $u^{k+1}$.

Step 2. Stopping test. If $\left\|u^{k+1}-u^{k}\right\| \leq \varepsilon_{1}$ where $\varepsilon_{1} \in \mathbb{R}_{++}^{1}$ is a preset tolerance, stop and declare $u^{*} \approx u^{k+1}$. Otherwise set $k=k+1$ and and go to Step 1 .

It is not hard to prove that the fixed point algorithm converges when we have Lipschitz continuity and strong monotonicity of the effective delay operators. Such a proof is presented in Friesz (2010), and in Section 7 for a special case of (A.35)-(A.38).

\section{Appendix A.7. Mathematical Programming Algorithms for Optimal Control}

The solution of optimal control problems in continuous time may be approached using algorithms familiar from finite dimensional nonlinear programming. To illustrate this point, we will be concerned with constructing an algorithm for the constrained infinite-dimensional mathematical program:

$$
\min J(u) \quad \text { s.t. } u \in U \subset V
$$

where $V$ is a Hilbert space and

$$
J: V \longrightarrow \mathbb{R}_{+}^{1}
$$

Furthermore, we suppose that $J(u)$ is G-differentiable on $U$ and that $V=\left(L^{2}\left[t_{0}, t_{f}\right]\right)^{m}$. We take $V$ to be a Hilbert space and the G-derivative of the functional $J(u)$ to be well defined. So we may immediately articulate a variational inequality for the optimal solution $u^{*} \in U$ :

$$
\delta J\left(u^{*}, \phi\right)=\left\langle\nabla J\left(u^{*}\right), u-u^{*}\right\rangle \geq 0 \quad \forall u \in U
$$

Appendix A.7.1. The Minimum Norm Projection

Consider the mathematical program

$$
\min \left\|u-u^{k}\right\| \quad \text { s.t. } u \in U \subset V
$$

where $V$ is a Hilbert space and $\|v\|=\left\langle v^{T}, v\right\rangle^{\frac{1}{2}}$ denotes the norm induced by the scalar product. We say that

$$
z^{k}=P_{U}\left[u-u^{k}\right]=\underset{u \in U}{\operatorname{argmin}}\left\|u-u^{k}\right\|
$$

is the minimum norm projection of $u^{k}$ onto the convex and closed subset $U$. It is equivalent to write 


$$
\begin{aligned}
z^{k} & =\underset{u \in U}{\operatorname{argmin}} \frac{1}{2}\left\|u-u^{k}\right\|^{2} \\
& =\underset{u \in U}{\operatorname{argmin}}\left[J_{\operatorname{proj}}^{k}(u)=\frac{1}{2}\left(u-u^{k}\right)^{T} \cdot\left(u-u^{k}\right)\right] \\
& \equiv \underset{u \in U}{\operatorname{argmin}} \int_{0}^{1} \frac{1}{2}\left(u-u^{k}\right)^{2} d t
\end{aligned}
$$

since squaring the norm is a monotonic transformation.

Appendix A.7.2. Structure of the Gradient Projection Algorithm

The gradient projection algorithm is summarized by the following updating rule:

$$
u^{k+1}=P_{U}\left[u^{k}-\theta_{k} \nabla J\left(u^{k}\right)\right]
$$

where the superscript $k$ denotes an iteration index and $\theta_{k}$ is a step size for iteration $k$. Note that this is fundamentally the same projection algorithm familiar from finite-dimensional mathematical programming, but it is now carried out in a Hilbert space $V$ of which $U$ is a subset; the mechanics of the projection are governed by the variational inequality (A.12).

Based on (A.44) we may now provide the following formal statement of the gradient projection algorithm:

\section{Gradient Projection Algorithm}

Step 0. Initialization. Set $k=0$ and pick $u^{0}(t) \in\left(L^{2}\left[t_{0}, t_{f}\right]\right)^{m}$.

Step 1. Find state trajectory. Using $u^{k}(t)$ solve the state initial-value problem

$$
\begin{aligned}
\frac{d x}{d t} & =f\left(x, u^{k}, t\right) \\
x\left(t_{0}\right) & =x_{0}
\end{aligned}
$$

and call the solution $x^{k}(t)$.

Step 2. Find adjoint trajectory. Using $u^{k}(t)$ and $x^{k}(t)$ solve the adjoint final value problem

$$
\begin{aligned}
(-1) \frac{d \lambda}{d t} & =\frac{\partial H\left(x^{k}, u^{k}, \lambda, t\right)}{\partial x} \\
\lambda\left(t_{f}\right) & =\frac{\partial K\left[x\left(t_{f}\right), t_{f}\right]}{\partial x}
\end{aligned}
$$

and call the solution $\lambda^{k}(t)$.

Step 3. Find gradient. Using $u^{k}(t), x^{k}(t)$, and $\lambda^{k}(t)$ calculate

$$
\begin{aligned}
\nabla_{u} J\left(u^{k}\right) & =\frac{\partial H\left(x^{k}, u^{k}, \lambda, t\right)}{\partial u} \\
& =\frac{\partial f_{0}\left(x^{k}, u^{k}, t\right)}{\partial u}+\left(\lambda^{k}\right)^{T} \frac{\partial f\left(x^{k}, u^{k}, t\right)}{\partial u}
\end{aligned}
$$


Step 4. Update and apply stopping test. For a suitably small step size $\theta_{k}$, update according to

$$
u^{k+1}=P_{U}\left[u^{k}-\theta_{k} \nabla J\left(u^{k}\right)\right]
$$

If an appropriate stopping test is satisfied, declare

$$
u^{*}(t) \approx u^{k+1}(t)
$$

Otherwise set $k=k+1$ and go to Step 1 .

\section{References}

Ban, X. J., Liu, H. X., Ferris, M. C., Ran, B. (2008). A link-node complementarity model and solution algorithm for dynamic user equilibria with exact flow propagations. Transportation Research Part B, 42(9), 823-842.

Ban, X., Pang, J.S., Liu, H.X., Ma, R. (2012). Modeling and solving continuous-time instantaneous dynamic user equilibria: a differential complementarity systems approach. Transportation Research Part B, 46, 389-408.

Bernstein, D., Friesz, T.L. and Tobin, R.L., 1993. A variational control formulation of the simultaneous route and departure-time choice equilibrium problem. In International Symposium on the Theory of Traffic Flow and Transportation, 12th, 1993, 107-126. Berkeley, California, USA.

Bliemer, M.C. and Bovy, P.H. (2003). Quasi-variational inequality formulation of the multiclass dynamic traffic assignment problem. Transportation Research Part B, 37(6), pp.501-519.

Browder, F.E., 1968. The fixed point theory of multi-valued mappings in topological vector spaces. Mathematische Annalen 177, $283-301$.

Camlibel, M.K., 2001. Complementarity methods in the analysis of piecewise linear dynamical systems, Ph.D. thesis, Center for Economic Research, Tilburg University, the Netherlands.

Camlibel, M.K., Pang, J.S., Shen, J.L., 2006a Conewise linear systems, non-Zenoness and observability. SIAM Journal on Control and Optimization 45, 1769-1800.

Camlibel, M.K., Pang, J.S., Shen, J.L., 2006b. Lyapunov stability of complementarity and extended systems. SIAM Journal on Optimization 17, 1056-1101.

Camlibel, M.K., Heemels, W.P.M.H., Schumacher, J.M., 2008 Algebraic necessary and sufficient conditions for the controllability of conewise linear systems. IEEE Transactions on Automatic Control 53, 762-774.

Chiu, Y.C., Bottom, J., Mahut, M., Paz, A., Balakrishna, R., Waller, T. and Hicks, J., 2011. Dynamic traffic assignment: A primer. Transportation Research E-Circular, (E-C153).

Friesz, T. L., Bernstein, D., Smith, T. E., Tobin, R. L., and Wie, B. W. (1993). A variational inequality formulation of the dynamic network user equilibrium problem. Operations Research, 41(1), 179-191.

Friesz, T. L., Bernstein, D., Suo, Z., and Tobin, R. L. (2001). Dynamic network user equilibrium with state-dependent time lags. Networks and Spatial Economics, 1(3), 319-347.

Friesz, T. L., and Mookherjee, R. (2006). Solving the dynamic network user equilibrium problem with state-dependent time shifts. Transportation Research Part B, 40(3), 207-229.

Friesz, T.L., Mookherjee, R., Rigdon, M. A., 2004. Differential variational inequalities with controls and state-dependent time shifts and their application to differential games. In Proceedings of the 11th International Symposium on Dynamic Games and Applications, 90-109, Tucson, Arizona.

Friesz, T. L., Rigdon, M. A., and Mookherjee, R. (2006). Differential variational inequalities and shipper dynamic oligopolistic network competition. Transportation Research Part B: Methodological, 40(6), 480-503.

Friesz, T. L., Kwon, C., and Mookherjee, R. (2007a). A computable theory of dynamic congestion pricing. Transportation and traffic theory, 1-26.

Friesz, T. L., Mookherjee, R., and Rigdon, M. A. (2007b). Formulating and solving service network pricing and resource allocation games as differential variational inequalities. Annals of the International Society of Dynamic Games: Advances in Dynamical Games (S. Jorgensen, M. Quincampoix and T. L. Vincent, editors), 9, 587-614, Birkhäuser, 2006.

Friesz, T. L., Mookherjee, R., Holguín-Veras, J., and Rigdon, M. A. (2008). Dynamic pricing in an urban freight environment. Transportation Research Part B, 42(4), 305-324.

Friesz, T.L. (2010). Dynamic Optimization and Differential Games, Springer, New York.

Friesz, T. L., Kim, T., Kwon, C., and Rigdon, M. A. (2011). Approximate network loading and dual-time-scale dynamic user equilibrium. Transportation Research Part B, 45(1), 176-207.

Friesz, T. L., Han, K., Neto, P. A., Meimand, A., and Yao, T. (2013). Dynamic user equilibrium based on a hydrodynamic model. Transportation Research Part B, 47, 102-126.

Friesz, T.L. and Meimand, A. (2014). A differential variational inequality formulation of dynamic network user equilibrium with elastic demand. Transportmetrica A: Transport Science, 10(7), 661-668.

Han, K., Friesz, T.L., Yao, T. (2013a). A partial differential equation formulation of Vickrey's bottleneck model, part I: Methodology and theoretical analysis. Transportation Research Part B 49, 55-74.

Han, K., Friesz, T.L., Yao, T. (2013b). A partial differential equation formulation of Vickrey's bottleneck model, part II: Numerical analysis and computation. Transportation Research Part B, 49, 75-93. 
Han, K, Friesz, TL, Yao, T, 2013c. Existence of simultaneous route and departure choice dynamic user equilibrium. Transportation Research Part B, 53, 17-30.

Han, K., Friesz, T.L., Szeto, W.Y., Liu H. (2015a). Elastic demand dynamic network user equilibrium: Formulation, existence and computation, Transportation Research Part B, 81, 183-209.

Han, K., Szeto, W.Y., Friesz, T.L. (2015b). Formulation, existence, and computation of boundedly rational dynamic user equilibrium with fixed or endogenous user tolerance, Transportation Research Part B, 79, 16-49.

Han, K., Piccoli, B., Szeto, W.Y. (2016). Continuous-time link-based kinematic wave model: formulation, solution existence, and well-posedness. Transportmetrica B: Transport Dynamics, 4 (3), 187-222.

Heemels, W. P.H. , 1999. Linear complementarity systems: a study in hybrid dynamics, Ph.D. thesis, Department of Electrical Engineering, Eindhoven University of Technology, the Netherlands.

Hipfel, D., 1993. The nonlinear differential complementarity problem, Ph.D. thesis, Department of Mathematical Sciences, Rensselaer Polytechnic Institute, the USA.

Kachani, S., and Perakis, G. (2009). A dynamic travel time model for spillback. Networks and Spatial Economics, 9(4), 595.

Lo, H., Szeto, W. (2002). A cell-based variational inequality formulation of the dynamic user optimal assignment problem. Transportation Research Part B 36 (5), 421-443.

Mookherjee, R., and Friesz, T.L. (2008). Pricing, allocation, and overbooking in dynamic service network competition when demand is uncertain. Production and Operations Management, 17(4), 455-474.

Pang, J. S., and Stewart, D. E. (2008). Differential variational inequalities. Mathematical Programming, 113(2), 345-424.

Perakis, G., and Roels, G. (2006). An analytical model for traffic delays and the dynamic user equilibrium problem. Operations Research, 54(6), 1151-1171.

Peeta, S. and Ziliaskopoulos, A.K., 2001. Foundations of dynamic traffic assignment: The past, the present and the future. Networks and Spatial Economics, 1(3-4), pp.233-265.

Ran, B., and Boyce, D. E. (1996). Modelling dynamic transportation networks: an intelligent transportation system oriented approach, Springer, 356 pp.

Ran, B., Boyce, D. E., and LeBlanc, L. J. (1993). A new class of instantaneous dynamic user-optimal traffic assignment models. Operations Research, 41(1), 192-202.

Ran, B., Hall, R.W. and Boyce, D.E. (1996). A link-based variational inequality model for dynamic departure time/route choice. Transportation Research Part B, 30(1), 31-46.

Rudin, W., 2006. Functional Analysis. McGraw-Hill.

Szeto, W.Y. and Lo, H.K. (2004). A cell-based simultaneous route and departure time choice model with elastic demand. Transportation Research Part B, 38(7), pp.593-612.

Szeto, W.Y. and Lo, H.K., 2005. Dynamic Traffic assignment: review and future research directions. Journal of Transportation Systems Engineering and Information Technology, 5(5), pp.85-100.

Vickrey, W.S. (1969). Congestion Theory and Transport Investment. The American Economic Review, 59 (2), $251-261$.

Yperman, I., S. Logghe and L. Immers (2005). The Link Transmission Model: An Efficient Implementation of the Kinematic Wave Theory in Traffic Networks, Advanced OR and AI Methods in Transportation, Proc. 10th EWGT Meeting and 16th Mini-EURO Conference, Poznan, Poland, 122-127, Publishing House of Poznan University of Technology.

Zhu, D., and Marcotte, P. (2000). On the existence of solutions to the dynamic user equilibrium problem. Transportation Science, 34(4), 402-414. 